\title{
Application of selective polymeric sorbents for simple coumarins extraction from deodorant samples
}

\author{
Katarína Hroboňová, Andrea Špačková \\ Slovak University of Technology in Bratislava, Faculty of Chemical and Food Technology, \\ Institute of Analytical Chemistry, Radlinského 9, SK-812 37 Bratislava, Slovak Republic \\ katarina.hrobonova@stuba.sk
}

\begin{abstract}
Coumarins (2H-1-benzopyran-2-coumarin derivatives) are derivatives of cinnamic acid naturally occurring in many plants, fungi, and fruits. They are used as ingredients in cosmetics to enhance the aroma and other biological effects. In this work, cosmetic samples (deodorants) were treated by solid phase extraction prior to high performance liquid chromatography determination of coumarins. Traditional sorbent (C18) and selective polymer-based sorbents (laboratory prepared and commercial) were used for solid phase extraction. Recovery values were above $85 \%$ (RSDs below $6 \%$ ) except for esculin, where the recovery was lower. Core-shell column of C18 type and gradient of mobile phase methanol-1 \% acetic acid were used for high performance liquid chromatography analysis of extracts. Limits of quantitation were $0.5 \mu \mathrm{g} \mathrm{mL^{-1 }}$ for coumarin (ultraviolet detection) and below $12 \mathrm{ng} \mathrm{mL}^{-1}$ for esculin, umbelliferonene, scoparone, 4-methylumbelliferone, herniarin (fluorescence detection). In the tested samples, no coumarins were detected.
\end{abstract}

Keywords: simple coumarins, deodorants, high performance liquid chromatography, selective sorbent, solid phase extraction

\section{Introduction}

Coumarins (Fig. 1) are substances naturally occurring in plants (e.g. tonka bean, yellow sweet clover, lavender), and other natural sources. They exhibit several biological effects with a wide range of applications, e.g. UV protection, antibacterial, nematocidal, phytotoxic, antifungal activity, etc. (Pan et al., 2014). Coumarin, as the main group representative, is characterized by its smell which is sweet, from herbal-spicy to slightly balsamic with coconut variations; in dilution it smells like freshly cut hay.

Fragrances are used extensively in cosmetics, perfumes and personal care products (aftershave lotions, bath products, bubble baths, cleansing products, moisturizers, skin care products and suntan products). Among coumarins, synthetic coumarin is the main compound utilized as fragrance ingredient in cosmetic products at concentrations from $0.08 \%$ to $5.8 \%$ (Quantitative Risk Assessment aggregate exposure adjusted upper concentration levels; Guidance for the use of IFRA standards, 2019). Some natural coumarins may be present in cosmetic preparations from plant extracts (Murray, 2002). In the EU, seven coumarins (herniarin, dicoumarol, 7-ethoxy-4-methylcoumarin, dihydrocoumarin, 7-methylcoumarin, acenocoumarol, and pyranocoumarin) are forbidden in cosmetic products (Regulation (EC) No 1223/2009). Coumarins not only improve the aroma of the products, but from a technological point of view they serve mainly as a modifier and fixator of the final fragrant composition. However, they can also cause allergic reactions (Srikrishna et al., 2018). A quantitative human health risk assessment integrating both cancer and non-cancer effects confirmed the safety of coumarin exposure from natural dietary sources as well as from its use as perfume in personal care products (Felter et al., 2006).

$\begin{array}{lcccc} & & & & \\ \text { Coumarin } & \mathrm{H} & \mathrm{H} & \mathrm{H} & \mathrm{H} \\ \text { Esculin } & \mathrm{H} & \mathrm{Glucose} & \mathrm{OH} & \mathrm{H} \\ \text { Umbelliferone } & \mathrm{H} & \mathrm{H} & \mathrm{OH} & \mathrm{H} \\ \text { 4-Methylumbelliferone } & \mathrm{CH}_{3} & \mathrm{H} & \mathrm{OH} & \mathrm{H} \\ \text { Scoparone } & \mathrm{H} & \mathrm{CH} & \mathrm{CH}_{3} \mathrm{O} & \mathrm{H} \\ \text { Herniarin } & \mathrm{H} & \mathrm{H} & \mathrm{CH}_{3} \mathrm{O} & \mathrm{H}\end{array}$

Fig. 1. Chemical structure of selected natural coumarins.

According to EU Council Directive, if fragrances concentration exceeds $100 \mathrm{ppm}$ in "wash-off" products such as shampoos and body wash, or $10 \mathrm{ppm}$ in "leave-on" products such as creams and perfumes, they need to be enumerated in the ingredients list 
of the product (EU Council Directive No 76/768/ EEC). Analytical methods that screen for these fragrance compounds in complex matrices have to be developed. HPLC is most commonly used method for separation and determination of coumarins, while GC and TLC are rarely used for separation of some coumarins (Waksmundzka-Hajnos et al., 2006; Rahim et al., 2011). The most common HPLC stationary phase is the C18, C8, phenyl or phenyl-hexyl type. A mixture of an organic solvent and aqueous acid solution with gradient elution is often chosen as the mobile phase. A spectrophotometric detector, fluorescent detector (Hroboňová et al., 2013) or a mass spectrometric detector (Ma et al., 2015) are used to detect coumarins. An important part of chemical analysis is the preparation of samples to remove interferences from the matrix and to obtain a fraction of the sample or the final analyte extract in a solvent compatible with the analytical technique used, e.g. with the mobile phase in HPLC. Sometimes it is also necessary to preconcentrate the target analytes. The most commonly used sample preparation technique is solid phase extraction (SPE) due to its speed, simplicity of procedures and equipment, automatization and the small amount of solvents required compared to traditional extraction techniques, e.g. liquid-liquid and liquid-solid extraction. There are many of SPE sorbents but in most procedures only a few types are used (e.g. C18). Their selectivity can be sometimes low, especially in case of complex samples, and the target analyte can co-elute with the matrix interferences. A new type of sorbents with increased selectivity for target analytes are the molecularly imprinted polymers (MIP), synthetic tailor-made materials with predefined selectivity for the target analyte or structurally related compounds based on specific interactions between the analyte and the binding sites on MIP. In most cases, the application of MIPs in cosmetics' analysis is mainly focused on the extraction of active ingredients such as parabens, antimicrobials, UV filters (Figuierido et al., 2016; Vicario et al., 2018; Wang et al., 2018), but they are also applied for selective extraction of toxic compounds, e.g. bisphenol (Zhu et al., 2010). MIP based sorbents are also useful in the analysis natural resources and extracts, e.g. herbal plant extracts, used in natural cosmetics, both for isolation and enrichment, active compounds such as alpha-lipoic acid (Xu et al., 2020) and toxins such as pyrrolizidine alkaloids (Luo et al., 2019). Only a few papers have been published regarding the application of MIPs selective for coumarins. MIPs have been used as sorbents for offline MIP-SPE extraction of coumarins from traditional Chinese medicine herbs, such as esculetin from Ash bark
(Hu et al., 2005) and esculin from Cortex fraxini (Wang et al., 2007). Coumarin, herniarin and umbelliferone were extracted from plants (lavender, archangel, camomile) macerates (Machyňáková and Hroboňová 2017a; Machyňáková and Hroboňová 2017b) and sesquiterpene coumarins from Asafoetida plant (Eidi et al., 2020).

In this study, extraction efficiency of simple coumarins obtained using different types of SPE sorbents were compared. The traditional sorbent of C18 type and selective polymer-based sorbents (laboratory synthesized and commercial) were tested. An offline extraction procedure and HPLC method were used for deodorant analysis.

\section{Material and Methods}

\section{Chemicals and samples}

Standards of coumarin (99\%), esculin (6,7-dihydroxycoumarin-6- $\beta$-D-glucoside, $98 \%$ ), umbelliferone (7-hydroxycoumarin, $99 \%$ ), 4-methylumbelliferone (4-methyl-7-hydroxycoumarin, $98 \%$ ), scoparone (6,7-dimethoxycoumarin, $98 \%$ ), herniarin (7-methoxycoumarin, $98 \%$ ) were purchased from Sigma-Aldrich (St. Louis, USA). Methanol (HPLC gradient grade) and acetic acid (99\%) were purchased from Merck (Darmstadt, Germany). Deionized water (resistivity of $18.2 \mathrm{M} \Omega / \mathrm{cm}$ ) was obtained from a AquaMax ultra (series 370) water purification system.

Samples of deodorants (Sample 1 was deodorant with the aroma of green tea and agave containing alcohol; Sample 2 was deodorant for sensitive skin without alcohol; Sample 3 was natural cosmetic deodorant where the manufacturer guarantees conscious renunciation of disputed ingredients) were obtained from a local drugstore network. For all tested samples, the manufacturers do not list coumarin as an ingredient in the product. The samples were kept at room temperature.

\section{Preparation of standard solutions}

Accurately weighed amounts of standards were dissolved in solvent (initial mobile phase methanol/ $1 \%$ acetic acid $(20 / 80, v / v)$ to reach stock solutions with the concentration of $0.1 \mathrm{mg} \mathrm{mL}^{-1}$. Mixed working solutions were prepared by diluting the stock solutions to concentrations in

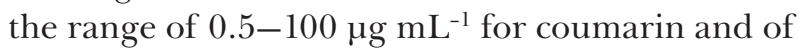
15-100 $\mathrm{ng} \mathrm{mL} \mathrm{mL}^{-1}$ for other substances under study.

\section{Solid phase extraction procedure}

SPE was performed with C18 Hydra (Chromabond, Macherey-Nagel, Germany; $100 \mathrm{mg}$ of sorbent), MIP-phenolics (AFFINIMIP, Affinisep, France; $100 \mathrm{mg}$ of sorbent), and MIP-coumarins (labora- 
tory prepared under procedure by Machyňáková and Hroboňová, 2017a; $100 \mathrm{mg}$ of sorbent). SPE cartridges were preconditioned with $2 \mathrm{~mL}$ of methanol and $2 \mathrm{~mL}$ of water. Then, $0.5 \mathrm{~mL}$ of sample or standard solution of coumarins was passed through the cartridge. The cartridge was washed with $1 \mathrm{~mL}$ of water and finally the analytes were eluted with $0.5 \mathrm{~mL}$ of methanol/acetic acid (9/1, v/v). This extract was filtered through a $0.45 \mu \mathrm{m}$ nylon membrane filter and an aliquot of $20 \mu \mathrm{L}$ was injected into the HPLC.

\section{HPLC analysis}

HPLC separation was carried out on an Agilent Technologies, series 1260, Liquid chromatographic system equipped with a binary pump, injector valve (Rheodyne), column thermostat, diode array detector, and a fluorescence detector. A Kinetex C18 analytical column $(100 \mathrm{~mm} \times 4.6 \mathrm{~mm}$ I.D., $5 \mu \mathrm{m}$ particle size) was employed. The chromatographic elution was performed with binary mobile phase gradient consisting of methanol/acetic acid (99/1, v/v) (A) and a $1 \%$ aqueous solution of acetic acid (B) at the flow rate of $1.0 \mathrm{~mL} \mathrm{~min}^{-1}$. The gradient program was as follows: initial gradient conditions were set to $20 \%$ A, then linear gradient was increased to $45 \%$ A over $12 \mathrm{~min}$. At $12 \mathrm{~min}$, the gradient was programmed to $100 \%$ A over $0.5 \mathrm{~min}$ and held for $2 \mathrm{~min}$. At $14.5 \mathrm{~min}$, the gradient was returned to initial conditions over $0.5 \mathrm{~min}$ and held for $4 \mathrm{~min}$. Column temperature was maintained at $23{ }^{\circ} \mathrm{C}$. Injection volume was $20 \mu \mathrm{L}$. DAD was operated in the wavelength range of 190-400 $\mathrm{nm}$ and for quantification of coumarin, detection at $280 \mathrm{~nm}$ was used (corresponding to the wavelength of absorption maximum of coumarin in appropriate mobile phase). Fluorescence detection of esculin, umbelliferone, 4-methylumbelliferone, scoparone, herniarin was operated at the wavelengths of $330 \mathrm{~nm}(\lambda \mathrm{ex})$ and $450 \mathrm{~nm}(\lambda \mathrm{em})$ (corresponding to the wavelengths of absorption/ emmision maximum of coumarins in appropriate mobile phase). Fluorescence spectra were scanned in the wavelength range of $340-500 \mathrm{~nm}$.

\section{Method validation}

Analytical evaluation of the HPLC method was investigated using standard solutions in concentration ranges from 0.5 to $100 \mu \mathrm{g} \mathrm{mL}^{-1}$ for coumarin and from 15 to $100 \mathrm{ng} \mathrm{mL}^{-1}$ for other substances under study (six concentration levels of each analyte, three replicate measurements for each solution). Calibration curve of the analyte was obtained by plotting a graph of mean peak area versus the corresponding analyte concentration. LODs of six analytes were estimated using $3 s_{\mathrm{a}}$ criteria and LOQs as $10 s_{\mathrm{a}}$ criteria ( $s_{\mathrm{a}}$ is the standard deviation of the intercept of the calibration curve). For recovery study, two aliquots of deodorant (sample 3) were spiked with coumarins stock solutions giving a concentrations of $2.0 \mu \mathrm{g} \mathrm{mL} \mathrm{m}^{-1}$ and $20 \mu \mathrm{g} \mathrm{mL} \mathrm{m}^{-1}$ of coumarin, $20 \mathrm{ng} \mathrm{mL}^{-1}$ and $50 \mathrm{ng} \mathrm{mL}^{-1}$ of esculin, umbelliferone, scoparone, 4-methylumbelliferone, herniarin. The spiked samples were vigorously vortexed for $1 \mathrm{~min}$ and subsequently treated by the SPE procedure with an MIP-coumarins sorbent. Recoveries were determined by comparing the peak areas obtained from sample spiked with analytes after the extraction with those from the reference solution. Precisions of the method was evaluated for six sample preparations within three days and was expressed as relative standard deviation (RSD \%).

\section{Results and Discussion}

\section{Selection of SPE sorbent}

Commercial SPE sorbents, C18 Hydra, MIP-phenolics and laboratory prepared MIP-coumarins were tested for coumarins extraction from deodorant samples. The extraction procedure was the same for all tested sorbents and includes optimal conditions, methanol and water as conditioning solvents, water as washing solvent, methanol/acetic acid as eluting solvent. Octadecyl silica sorbent is most frequently used for the isolation of less polar compounds. The main retention mechanism is based on van der Waals forces. Modified C18 Hydra sorbent enables the extraction of more polar analytes from water matrices. MIP-phenolics sorbent is suitable for the extraction, clean up, and preconcentration of phenolic compounds (information of sorbent producers). Laboratory prepared MIP was synthesized by thermal bulk polymerization using umbelliferone as the template. Optimization of polymerization (ratios and types of polymerization constituents) allows preparing sorbents with good morphology (evaluated by scanning electron microscopy), high specific adsorption capacity (270 $\mu \mathrm{g}$ of umbelliferone per $1 \mathrm{~g}$ of polymer), and group selectivity for structurally related simple coumarins (Machyňáková and Hroboňová, 2017a). MIP sorbents, contrary to the traditional SPE materials (silica-based sorbents), are characterized by higher selectivity and specificity to the target analyte, which leads to effective elimination of interferences and matrix effects.

Extraction efficiencies of SPE sorbents were evaluated for standard solution of coumarin at the con-

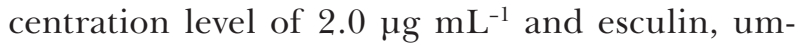
belliferone, scoparone, 4-methylumbelliferone, herniarin at the concentration level of $20 \mathrm{ng} \mathrm{mL}^{-1}$. Results of the recovery study are shown in Fig. 2. C18 type and MIP-phenolic sorbents showed 
nearly equal recovery for the investigated coumarins (87-97\%), except for esculin where the recovery reached values close to $60 \%$. Esculin is a molecule significantly different from other coumarins under study as it is a glucoside compound (Fig. 1). Thus, this more polar compound is weakly retained on the $\mathrm{C} 18$ sorbent which results in lover recovery. Comparing MIP based sorbents, the laboratory synthesized MIP-coumarins sorbent is suitable for the extraction of selected simple coumarins. Compared with other tested sorbents, higher recovery values $(90-97 \%$, RSDs $<6 \%$ ) were obtained for five aglycone coumarins. Also, higher recovery was achieved for esculin (72\%, RSD $=5 \%)$. Reduced recovery of esculin, compared to other analytes, on MIP-coumarins is related with the incompatibility of imprinted cavity with the shape and functionality of the analyte. MIP-phenolic, although they are more universal (imprinted cavity designed for phenolic compounds) are less selective for simple coumarins. Similar to MIP-coumarins, lower recovery for esculin was observed.

Average recovery of up to $74.7 \%$ was achieved for extraction and enrichment of esculin from Cortex fraxini plant using MIP sorbent prepared with MAA as the functional monomer and esculin as the template (Wang et al., 2007). Comparable recovery values as in the presented work were obtained for selective MIP-SPE extraction of umbelliferone and herniarin from chicory macerate (81.2-98.7 \%) (Machyňáková and Hroboňová, 2017a) as well as for extraction of coumarin, umbelliferone and herniarin from lavender, archangel and chamomile macerate (78.7-90.3\%, respectively) using MIP sorbent coated magnetite (Machyňáková and Hroboňová, 2017b).

A significant advantage of MIP based sorbents, in addition to selectivity for target analyte or analogues, is its reusability, which can be considered as green chemistry approach and it makes the analysis cheaper (Mariusz, 2019; Machyňáková and Hroboňová, 2017a). In this work, the MIP sorbents were reused more than five times for sample treatment (without the change of effectiveness by $5 \%$ ), versus only once for C18 type. One of the problems of MIP based sorbents are template residues present in the polymer matrix even after exhaustive washing steps. Leakage of the template can affect the accuracy of analyte determination. To overcome this problem, dummy MIP synthetic approach can be used. Eidi et al. (2020) synthesized MIP sorbent using umbelliferone as the template and it was used in SPE of structural analogues, galbanic acid, 7-isopentenyloxy coumarin and auraptene from aqueous plant macerate. The recovery was in the range of $68.3-84.7 \%$. (Eidi et al., 2020)

\section{Selection of chromatographic conditions}

HPLC conditions were selected after testing different columns and mobile phase compositions. The proportion of organic and aqueous phases as well as gradient profile were selected to provide efficient separation of esculin, umbelliferone, coumarin, scoparone, 4-methylumbelliferone and herniarin with a satisfactory run time, resolution higher than 1.5, good peak symmetry and minimal high equivalent theoretical plate. Optimization and evaluation of HPLC separation were performed using standard solution of coumarins. The core shell C18 type of stationary phase and the mobile phase consisting of methanol and water with an addition of $1 \%$ acetic acid in gradient elution

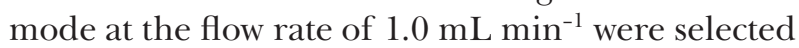
as the best chromatographic conditions. Figure $3 \mathrm{a}$ shows the chromatogram of a standard mixture and Table 1 documents relevant chromatographic characteristics obtained at the selected optimal conditions. The advantage of this type of column is the short total analysis time (about $15 \mathrm{~min}$ ) compared to columns with traditional silica based stationary phase (about $30 \mathrm{~min}$; Hroboňová et al.,

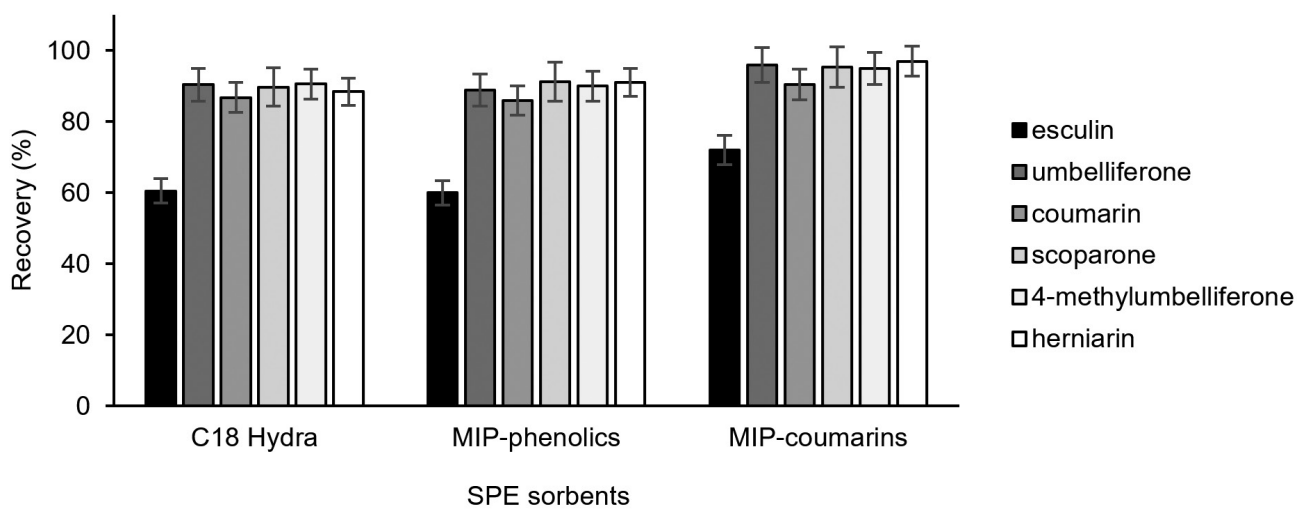

Fig. 2. Recovery of coumarins obtained for C18-Hydra, MIP-phenolics and MIP-coumarins SPE sorbents. 
Tab. 1. Chromatographic characteristics (elution time $\left(t_{\mathrm{R}}\right)$, resolution $\left(R_{\mathrm{s}}\right)$, high equivalent to a theoretical plate $(H)$ ) and reproducibility of retention times and peak areas $(A)$ for HPLC separation of coumarins under optimal chromatographic conditions ${ }^{1}$.

\begin{tabular}{|c|c|c|c|c|c|}
\hline \multirow[t]{2}{*}{ Compound } & \multirow{2}{*}{$\begin{array}{c}t_{\mathrm{R}} \\
(\min )\end{array}$} & \multirow[t]{2}{*}{$R_{\mathrm{s}}$} & \multirow{2}{*}{$\begin{array}{c}H \\
(\mu \mathrm{m})\end{array}$} & \multicolumn{2}{|c|}{$\begin{array}{c}\text { Reproducibility } \\
\text { RSD (\%) }\end{array}$} \\
\hline & & & & $t_{R}$ & $A$ \\
\hline Esculin & 2.8 & 18.6 & 4.5 & 0.8 & 5.1 \\
\hline Umbelliferone & 7.1 & 7.9 & 7.2 & 0.4 & 4.8 \\
\hline Coumarin & 8.9 & 2.6 & 5.7 & 0.3 & 5.5 \\
\hline Scoparone & 9.4 & 1.8 & 3.6 & 0.3 & 5.5 \\
\hline 4-Methylumbelliferone & 9.9 & 7.6 & 3.6 & 0.3 & 4.5 \\
\hline Herniarin & 11.9 & & 3.4 & 0.2 & 4.5 \\
\hline
\end{tabular}

${ }^{1}$ esculin, umbelliferone, scoparone, 4-methylumbelliferone and herniarine - parameters for fluorescence detection $\left(\lambda_{\mathrm{ex}} / \lambda_{\mathrm{em}}=330 / 450 \mathrm{~nm}\right)$; coumarin - parameters for UV detection at $(\lambda=280 \mathrm{~nm})$.

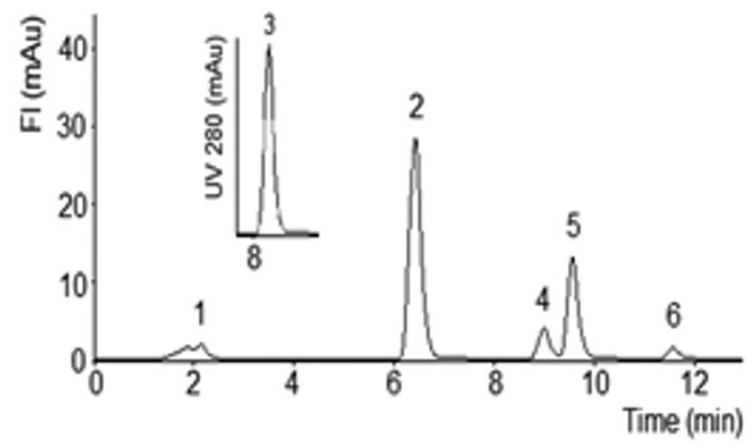

a)

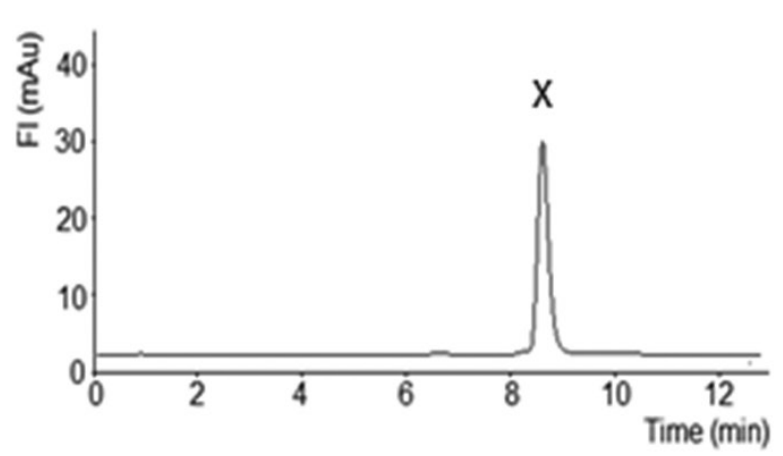

b)

Fig. 3. Chromatograms of standard mixture (a) and sample 2 extract after MIP-coumarins SPE (b). Chromatographic conditions: Kinetex C18 (100 mm $\times 4.6 \mathrm{~mm}$ I.D., $5 \mu \mathrm{m})$ column, gradient elution with methanol-1 \% acetic acid as mobile phase, flow rate of $1.0 \mathrm{~mL} \mathrm{~min}{ }^{-1}$, column temperature of $23{ }^{\circ} \mathrm{C}$, UV detection at $280 \mathrm{~nm}$, Fluorescence detection at $330 \mathrm{~nm}\left(\lambda_{\mathrm{ex}}\right)$ and $450 \mathrm{~nm}\left(\lambda_{\mathrm{em}}\right)$; legend: $1-$ esculin, 2 - umbelliferonene, 3 - comarin, 4 - scoparone, 5 - 4-methylumbelliferone, 6 - herniarin, $\mathrm{X}-$ unknown compound.

2013), which results from decreased core shell particles resistance to solute mass transfer into the porous structure of the stationary phase due to short diffusion distances (Hayes at al., 2014). For quantitative analysis, on-line coupled UV spectrophotometric and fluorescence detection were used. The UV detection wavelength was set to $280 \mathrm{~nm}$ for coumarin detection. The fluorescence excitation/ emission wavelengths were set to $330 / 450 \mathrm{~nm}$ for the detection of other coumarins under study, as it provides higher selectivity and sensitivity and less interference potential compared to UV detection.

\section{Method validation}

The developed method was validated in terms of linearity, LOQs, recoveries, and intra-day precision showing good linearity in the calibration

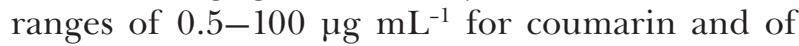
15-100 ng $\mathrm{mL}^{-1}$ for other coumarins; with correlation coefficients above 0.99 . LODs and LOQs of the six analytes are summarized in Table 2. The LOQs were found to be in the range of $1-12 \mathrm{ng} \mathrm{mL}{ }^{-1}$ for esculin, umbelliferone, scoparone, 4-methylumbelliferone and herniarin and $0.5 \mu \mathrm{g} \mathrm{mL} \mathrm{m}^{-1}$ for coumarin. The European Cosmetics Regulation prescribes to declare coumarin content from the concentration of $0.001 \%$ for leave-on and of $0.01 \%$ for rinse-off products. Thus, with lower LOQs, the developed method is suitable for coumarin determination (for other derivatives, there are no limits) in deodorants and antiperspirants of all types including fragranced body sprays (Guidance for the use of IFRA standards, 2019; EU Council Directive No 76/768/EEC). LOQs of coumarins in this study was lower than those reported in literature (32-45 $\mathrm{ng} \mathrm{mL}^{-1}$ for simple coumarins by HPLCDAD (Xiongfeng et al., 2016); $0.17 \mathrm{mg} \mathrm{kg}^{-1}$ for 6-methylcoumarin by HPLC-DAD, $5.0 \mathrm{mg} \mathrm{kg}^{-1}$ by GC-FID (Liu et al., 2018)), although the LOQs obtained by fluorescence detection are slightly higher 
Tab. 2. Linearity, LOQs, recoveries, inter-day precision of six coumarins.

\begin{tabular}{lcccccc}
\hline Compound & Linearity & $\boldsymbol{R}^{\mathbf{2}}$ & LOD $^{\mathbf{1}}$ & LOQ $^{\mathbf{1}}$ & $\begin{array}{c}\text { Recovery } \\
\text { (\%) }\end{array}$ & $\begin{array}{c}\boldsymbol{R S D} \\
(\%)\end{array}$ \\
\hline Esculin & $10-100$ & 0.987 & 3 & 10 & $70 / 72$ & $4.2 / 5.4$ \\
Umbelliferone & $1-100$ & 0.998 & 0.3 & 1 & $93 / 95$ & $4.5 / 5.0$ \\
Coumarin & $0.5-100$ & 0.995 & 0.2 & 0.5 & $89 / 90$ & $5.4 / 5.9$ \\
Scoparone & $10-100$ & 0.998 & 3 & 10 & $93 / 95$ & $4.1 / 5.2$ \\
4-Methylumbelliferone & $3-100$ & 0.997 & 1 & 3 & $92 / 95$ & $4.9 / 5.4$ \\
Herniarin & $12-100$ & 0.998 & 4 & 12 & $95 / 97$ & $6.4 / 6.4$ \\
\hline
\end{tabular}

${ }^{1} \mathrm{ng} \mathrm{mL}{ }^{-1}$ for esculin, umbelliferone, scoparone, 4-methylumbelliferone and herniarin; $\mu \mathrm{g} \mathrm{mL}^{-1}$ for coumarin;

${ }^{2}$ spiked concentrations: $2.0 \mu \mathrm{g} \mathrm{mL}^{-1} / 20 \mu \mathrm{g} \mathrm{mL}^{-1}$ of coumarin, $20 \mathrm{ng} \mathrm{mL}^{-1} / 50 \mathrm{ng} \mathrm{mL}^{-1}$ of esculin, umbelliferone, scoparone, 4-methylumbelliferone, herniarin.

than that obtained by the HPLC-MS/MS methods $\left(0.5-2 \mathrm{ng} \mathrm{mL} \mathrm{m}^{-1}\right.$ for 22 coumarin derivatives (Ma et al., 2015)). The recovery values of $70-97 \%$ were obtained (RSD \% 4.1-6.4) for sample 3 spiked with two substances concentration levels, confirming the applicability and good precision of the presented method.

\section{Samples analysis}

Practical applicability of the proposed method with MIP based SPE extraction was demonstrated on deodorant analysis. Sensitivity and selectivity of the developed method were found to be sufficient for the characterization of the six coumarins under study in samples. A representative chromatogram of the deodorant extract obtained by MIP-coumarins $\mathrm{SPE}$ is shown in Fig. 3b. No peaks were observed in the elution times of target coumarins, indicating that there were not interferences from other constituents of the sample. The results show that MIP sorbents are suitable for the extraction of substances from complex samples, mainly for cleaning and/or preconcentration of analytes. The sample preparation method in this study is efficient for the extraction of esculin, umbelliferone, coumarin, scoparone, 4-methylumbelliferone and herniarin from deodorants. For the tested samples, manufacturers do not specify the presence of synthetic coumarin, which does not exclude the presence of natural coumarins from plant extracts used in the preparation of the products. Based on HPLC analysis, coumarin nor its derivatives were detected at concentration levels over LOD in the tested deodorant samples.

\section{Conclusions}

In this study, laboratory prepared polymeric sorbent based on molecularly imprinted polymer reached nearly equal recovery for investigated coumarins compared to the C18 type and MIP-phenolics SPE sorbents. The advantage of the applied
MIP sorbent was its reuse (more than five times for sample pretreatment; C18 type can be used only once). Higher recovery was achieved for esculin extraction, a less hydrophobic compound under study, compared to the C18 sorbent. The method based on SPE using MIP-coumarins as the selective adsorbent shoved good recovery and precision. The HPLC method with UV spectrophotometric and fluorescence detection was found to be suitable for the separation and determination of coumarins in cosmetic products within a short time (sample preparation below $10 \mathrm{~min}$, chromatography of $15 \mathrm{~min}$ ). After some modification, this method can be extended for the analysis of various cosmetic or other complex samples.

\section{Acknowledgement}

This research was financially supported by the Scientific Grant Agency of the Ministry of Education of the Slovak Republic and the Slovak Academy of Sciences (grant No. VEGA 1/0159/20).

\section{References}

Eidi S, Iranshahi M, Mohammadinejad A, Mohsenzadeh MS, Farhadi F, Mohajeri SA (2020) J. Chromatogr. B 1138: 121943.

EU Council Directive of 27 July 1976 on the approximation of the laws of the Member States relating to cosmetic products (No 76/768/EEC) (OJ L 262, 27. 9. 1976, p. 169).

Felter SP, Vassallo JD, Carlton BD, Daston GP (2006) Food Chem. Toxicol. 44: 462-475.

Figuierido L, Erny GL, Santos L, Alves A (2016) Talanta 146: 754-765.

Guidance for the use of IFRA standards, The International Fragrance Association, December 12, 2019.

Hayes R, Ahmed A, Edge T, Zhang H (2014) J. Chromatogr. A 1357: 36-52.

Hroboňová K, Lehotay J, Čižmárok J, Sádecká J (2013) J. Liq. Chromatogr. Rel. Technol. 36: 486-503.

Hu SG, Li L, He XW (2005) J. Chromatogr. A 1062(1): 31-37.

Liu H, Zhang J, Hu B, Wei L (2018) China Surfactant Detergent and Cosmetics 48: 419-422. 
Luo Z, Chen G, Li X, Wang L, Shu H, Cui X, Chang C, Zeng A, Fu Q (2019) J. Sep. Sci. 42(21): 3352-3362.

Ma Q, Xi H, Ma H, Meng X, Wang Z, Bai H, Li W, Wang C (2015) Chromatographia 78: 241-249.

Machyňáková A, Hroboňová K (2017a) Chromatographia 80(7): 1015-1024.

Machyňáková A, Hroboňová K (2017b) Anal. Methods 9: 2168-2176.

Mariusz M (2019) Mip Synthesis, Characteristics and Analytical Application, in Comprehensive Analytical Chemistry, vol 86. Elsevier, Amsterdam.

Murray RDH (2002) The Naturally Occurring Coumarins. In: Herz W., Falk H., Kirby G.W., Moore R.E. (eds) Fortschritte der Chemie organischer Naturstoffe / Progress in the Chemistry of Organic Natural Products, vol 83. Springer, Vienna.

Pan TL, Wang PW, Aljuffali IA, Leu YL, Hung YY, Fang JY (2014) Toxicol. Lett. 226: 173-181.

Rahim AA, Saad B, Osman H, Yahya SA (2011) Food Chem. 126: 1412-1416.

Regulation (EC) No 1223/2009 of the European Parliament and of the Council of 30 November
2009 on Cosmetic Products (OJ L 342, 22. 12. 2009, p. 59) Official Journal of the European Union.

Srikrishna D, Godugu C, Dubey PK (2018) Mini Reviews in Medicinal Chemistry 18(2): 113-141.

Vicario A, Solari M, Felici E, Aragón L, Bertolino F, Gomez MR (2018) Microchem. J. 142: 329-334.

Wakssmundzka-Hajnos M, Petruczynik A, Hajnos M, Tuzimski T, Hawryl A, Bogucka-Kocka A (2006) J. Chrom. Sci. 44: 510-517.

Wang GS, Cao QE, Ding ZT, Wang YG, Yang MH (2007) Helv. Chim. Acta 90(6): 1179-1189.

Wang F, Li X, Li J, Zhu C, Liu M, Wu Z, Liu L, Tan X, Lei F (2018) J. Colloid Interf. Sci. 527: 124-131.

Xiongfeng H, Lvye L, Qun X, Rogrer J (2016) Thermo Scientific Application Note 1128: Determination of coumarins in cosmetics.

Xu L, Zhao ZX, Huang YA, Zhu QJ (2020) Molecules 25(312): $1-15$.

Zhu R, Zhao W, Zhai M, Wei F, Cai Z, Sheng N, Hu Q (2010) Anal. Chim. Acta 658: 209-216. 